\title{
Correction: Hydrocephalus is a rare outcome in community-acquired bacterial meningitis in adults: a retrospective analysis
}

\author{
Jacob Bodilsen ${ }^{*}$, Henrik Carl Schønheyder ${ }^{2}$ and Henrik Nielsen ${ }^{1}$
}

\section{Correction}

After publication of this work [1], we became aware of two errors in Table two (Table 1 here). In the column describing scanned patients without hydrocephalus the CSF:blood glucose index and CSF protein levels were incorrect and should instead be 0.04 and $4.6 \mathrm{~g} / \mathrm{L}$ respectively.

Table 1 Clinical and laboratory features of bacterial meningitis patients with and without hydrocephalus

\begin{tabular}{|c|c|c|}
\hline Characteristics $^{\mathrm{a}}$ & $\begin{array}{l}\text { Patients with } \\
\text { hydrocephalus } \\
(n=5)\end{array}$ & $\begin{array}{c}\text { Scanned patients } \\
\text { without } \\
\text { hydrocephalus } \\
(n=115)\end{array}$ \\
\hline Symptom duration (days) & 3.5 & 2 \\
\hline Headache & 3 & 66 \\
\hline Nausea & 2 & 54 \\
\hline Confusion & 4 & 114 \\
\hline \multicolumn{3}{|l|}{$\mathrm{GCS}^{\mathrm{b}}$} \\
\hline$<9$ & 1 & 19 \\
\hline $9-12$ & 1 & 49 \\
\hline$>12$ & 3 & 47 \\
\hline MAP $(\mathrm{mm} \mathrm{Hg})$ & 104 & N/A \\
\hline ICP $(\mathrm{mm} \mathrm{Hg})$ & - & N/A \\
\hline Heart rate (beats/min) & 85 & 100 \\
\hline $\mathrm{CRP}(\mathrm{mg} / \mathrm{L})$ & 293 & 232 \\
\hline Cranial nerve paresis & 2 & 22 \\
\hline $\begin{array}{l}\text { Sensory / motornerve } \\
\text { deficit }\end{array}$ & 0 & 11 \\
\hline $\begin{array}{l}\text { Seizures before/during } \\
\text { admission }\end{array}$ & 0 & 14 \\
\hline
\end{tabular}

* Correspondence: jacob.bodilsen@rn.dk

'Department of Infectious Diseases, Aalborg Hospital, Aarhus University Hospital, Hobrovej 18-22, PO box 365, 9100, Aalborg, Denmark
Table 1 Clinical and laboratory features of bacterial meningitis patients with and without hydrocephalus (Continued)

Characteristics $^{\mathrm{a}}$

Patients with hydrocephalus

Scanned patients without

hydrocephalus

\begin{tabular}{lcc} 
& $(\mathbf{n}=\mathbf{5})$ & $(\mathbf{n}=\mathbf{1 1 5})$ \\
\hline CSF leukocytes $\left(\times 10 \wedge^{6} / L\right)$ & 2766 & 1702
\end{tabular}

Polymorphonuclear

84

92

leukocytes (\%)

CSF erythrocytes $\left(x 10 \wedge^{6} / L\right)$

$115 \quad 140$

CSF:blood glucose index

140

CSF protein $(g / L)$

0.005

0.04

Positive CSF culture

5.9

4.6

5

103

Positive blood culture

$$
2
$$

Antibiotics

$$
\begin{aligned}
& \text { Day } 1 \\
& \text { Day } 2 \\
& \text { After day } 2
\end{aligned}
$$

$$
4
$$

Dexamethasone $^{d}$

GOS $^{\mathrm{e}}$

\section{Unfavourable \\ outcome (GOS 1-4)}

Favourable outcome

(GOS 5)

Length of stay (days)

${ }^{a}$ Median values are used for continuous variables.

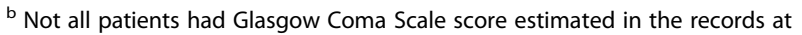
admission but could be assessed to have a score $<9,9-12$ or $>12$ based upon data review.

' One patient never scanned was not given appropriate antibiotic therapy during admission for unclear reasons.

${ }^{d}$ According to meningitis guidelines.

e Glasgow Outcome Scale: 1. Death, 2. Vegetative state, 3. Dependent upon others in daily life, 4 . Sequelae, but independent life, 5 . No or only minor sequelae.

Abbreviations: CSF Cerebrospinal fluid. GCS Glasgow coma scale. GOS Glasgow outcome scale. ICP Intracranial pressure. MAP Mean arterial pressure. N/A Not available. 
We apologize if this has caused any inconvenience to readers of the article.

\section{Author details}

'Department of Infectious Diseases, Aalborg Hospital, Aarhus University Hospital, Hobrovej 18-22, PO box 365, 9100, Aalborg, Denmark. ²Department of Clinical Microbiology, Aalborg Hospital, Aarhus University Hospital, Aarhus, Denmark.

Received: 8 November 2013 Accepted: 13 November 2013

Published: 19 November 2013

\section{Reference}

1. Bodilsen J, Schønheyder HC, Nielsen HI: Hydrocephalus is a rare outcome in community-acquired bacterial meningitis in adults: a retrospective analysis. BMC Infect Dis 2013, 13:321.

doi:10.1186/1471-2334-13-553

Cite this article as: Bodilsen et al:: Correction: Hydrocephalus is a rare outcome in community-acquired bacterial meningitis in adults: a retrospective analysis. BMC Infectious Diseases 2013 13:553.

\section{Submit your next manuscript to BioMed Central and take full advantage of:}

- Convenient online submission

- Thorough peer review

- No space constraints or color figure charges

- Immediate publication on acceptance

- Inclusion in PubMed, CAS, Scopus and Google Scholar

- Research which is freely available for redistribution 